\title{
Miller, Tilly: Konstruktivismus und Systemtheorie (Soziale Arbeit und ihre erkenntnistheoretischen Zugänge, Bd. 7), 130 S., Beltz Juventa, Weinheim 2021.
}

\author{
Lina Schröder
}

Angenommen: 30. Juni 2021 / Online publiziert: 9. Juli 2021

(C) Der/die Autor(en) 2021

Es ist kein Geheimnis, dass sich Historiker_innen gerne am Theorie- und Methodenangebot anderer Disziplinen, insbesondere bei der Soziologie, bedienen. Diesbezügliche solide Grundkenntnisse sollten entsprechend bereits während des Geschichtsstudiums vermittelt werden, zumal die Begrifflichkeiten, die in diesem Kontext dann ebenso von anderen Fächern übernommen werden, in den jeweiligen Ursprungsfächern nicht selten eine völlig andere Bedeutung innehaben. Die unterschiedlichen Fachsprachen sind so auch für alle Seiten eines der größten Probleme, wenn es um den interdisziplinären Dialog geht - egal ob - wie hier aus geschichtswissenschaftlicher Perspektive gedacht - mit der Soziologie, Kunstgeschichte, Archäologie oder gar mit den Naturwissenschaften.

Umso hilfreicher erscheinen daher Publikationen, die sich dieser Schwierigkeit widmen und sich um eine Einführung in eine der eigenen Fachtheorien in einer einfachen und Übersicht schaffenden Sprache bemühen. Insbesondere Letztere, die Übersicht, ist hier besonders wichtig, da jede Theorie in der Regel auch in einen eigenen Fachdiskurs eingebettet ist, der die jeweiligen Konzepte in den Kontext des Faches stellt, sie bewertet, kritisiert oder weiterführt. Manche Theorien, so auch die hier besprochene Systemtheorie, sind außerdem derart umfassend, dass nicht in jeder Untersuchung automatisch alle ihre Bestandteile Anwendung finden müssen.

Eine solche einführende Publikation liegt hier für den radikalen sowie sozialen Konstruktivismus und die Systemtheorie von Niklas Luhmann vor, wobei sie sich in erster Linie an Studierende, Doktoranden und Interessierte der angewandten Wissenschaft ,Soziale Arbeit' richtet. Die fünf Kapitel spiegeln dies wider: Während sich die ersten drei mit dem Begriff der Wissenschaft (Kapitel 1), dem Konstruktivismus (Kapitel 2) und der Luhmann'schen Systemtheorie (Kapitel 3) beschäftigen,

Lina Schröder $(\bowtie)$

Universität Würzburg, Würzburg, Deutschland

E-Mail: lina.schroeder@uni-wuerzburg.de 
befassen sich die letzten Abschnitte mit der Bedeutung (Kapitel 4) und Anwendung (Kapitel 5) der beiden Theorien in der Sozialen Arbeit. Das Ziel der Reihe, und damit auch des vorliegenden Bandes, besteht darin, den Lesern_innen ein Navigationssystem an die Hand zu geben, welches dazu beitragen soll zu verstehen, ,auf welcher Grundlage Theorien modelliert werden und was den Kern von Einzeltheorien ausmacht“ (S. 9). Wenn die Baupläne verstanden werden, so die Professorin für Sozialarbeit/Sozialpädagogik und Politikwissenschaft, Tilly Miller, lassen sich Unterschiede oder Schnittstellen zu anderen Theorien viel einfacher erkennen (S. 9).

Für angehende Historiker_innen oder diejenigen, die sich noch einmal einen schnellen Überblick über beide Theorien verschaffen möchten, sind vor allem die ersten drei Kapitel interessant. Vom Erkenntnis- und Wissenschaftsbegriff ausgehend, nähert sich Miller über die verschiedenen Deutungsmöglichkeiten von SubjektObjekt-Beziehungen den Theorien an (S. 15f.). So gehen sowohl Konstruktivismus als auch Systemtheorie davon aus, dass das Subjekt die Welt an sich nicht erkennen kann. Stattdessen lässt sich das, was wahrgenommen wird, immer nur deuten und interpretieren sowie durch den eigenen subjektiven Filter konstruieren (S. 18). Jede Theorie hat diesbezüglich eigene Vorstellungen entwickelt.

Wichtige, auf den Fachdiskurs des radikalen Konstruktivismus Einfluss nehmende Akteure waren und sind so unter anderem Ernst von Glaserfeld, Heinz von Foerster, Jean Piaget, Humberto Maturana und Paul Watzlawick. Bedeutende Vertreter des sozialen Konstruktivismus sind hingegen Kenneth J. Gergen, Peter L. Berger, Thomas Luckmann sowie Michel Foucault mit seiner Diskursanalyse. Sie bemängeln an der radikalen Auslegung das Ignorieren kultureller Aspekte, indem sie propagieren, dass die Wahrnehmung von Wirklichkeit nicht lediglich auf der subjektiven Konstruktion beruhe, sondern auch an kulturelle Voraussetzungen geknüpft sei (S. 31).

Auch Luhmanns Gesellschaftstheorie ist eine Erkenntnistheorie. Ihre zentralen Begriffe (System, Umwelt, Struktur, Prozess, Funktion, Kontingenz, Komplexität, Sinn, Kommunikation) stehen in einem inneren Verweisungszusammenhang - eine hierarchische, nach Wichtigkeit systematisierte Ordnung ist unmöglich und nicht vorgesehen (S. 47). Jeder, der schon einmal versucht hat, einem anderen die Luhmann'sche Theorie vorzustellen, kennt so die Schwierigkeit des fehlenden Anfangs. Miller löst dieses Problem hier, indem sie dort beginnt, wo Luhmann selbst begonnen hat, bei Talcott Parsons' struktur-funktionalem Ansatz (S. 48). Dieser besagt, dass funktionale Leistungen erbracht werden müssen, um gewisse Systemstrukturen aufrecht erhalten zu können. Luhmann drehte diesen Denkansatz um, indem er danach fragt, welche Systemstrukturen gegeben sein müssen, um funktionale Leistungen aufrecht erhalten zu können (S. 49f.). Der Schwerpunkt von Millers Darstellung, auch hinsichtlich der zentralen Begriffe, liegt hier vor allem auf der funktional ausdifferenzierten Gesellschaft und der von Parsons übernommenen und weiterentwickelten Medientheorie. Am Ende der jeweiligen Kapitel verweist sie nicht nur auf die verschiedenen Kritikpunkte an beiden hier vorgestellten Theorien, sondern zeigt zugleich auch bestehende Zusammenhänge zwischen Beiden auf. Die letzten beiden Abschnitte sprechen, wie eingangs angemerkt, vor allem die an der Sozialen Arbeit Interessierten an, denn hier werden unter anderem Fachspezifka vorausgesetzt, die sich Historiker_innen erst erschließen müssen. 
Miller versteht Theoriearbeit innerhalb einer Disziplin als Jonglierarbeit. Die unterschiedlich großen und kleinen Bälle, so ihr Fazit, stehen dabei für die verschiedenen und immer wieder bezüglich ihrer Bedeutung wachsenden oder schrumpfenden Theorieangebote (S. 119). Mit dieser Metapher beschließt sie ihre gelungene Überblicksdarstellung genauso anschaulich, wie sie diese begonnen hat - an beiden Theorien interessierten Einsteigern_innen der Geschichtswissenschaft kann die Darstellung durchaus ans Herz gelegt werden.

Funding Open Access funding enabled and organized by Projekt DEAL.

Open Access Dieser Artikel wird unter der Creative Commons Namensnennung 4.0 International Lizenz veröffentlicht, welche die Nutzung, Vervielfältigung, Bearbeitung, Verbreitung und Wiedergabe in jeglichem Medium und Format erlaubt, sofern Sie den/die ursprünglichen Autor(en) und die Quelle ordnungsgemäß nennen, einen Link zur Creative Commons Lizenz beifügen und angeben, ob Änderungen vorgenommen wurden.

Die in diesem Artikel enthaltenen Bilder und sonstiges Drittmaterial unterliegen ebenfalls der genannten Creative Commons Lizenz, sofern sich aus der Abbildungslegende nichts anderes ergibt. Sofern das betreffende Material nicht unter der genannten Creative Commons Lizenz steht und die betreffende Handlung nicht nach gesetzlichen Vorschriften erlaubt ist, ist für die oben aufgeführten Weiterverwendungen des Materials die Einwilligung des jeweiligen Rechteinhabers einzuholen.

Weitere Details zur Lizenz entnehmen Sie bitte der Lizenzinformation auf http://creativecommons.org/ licenses/by/4.0/deed.de. 